\title{
Prémices à une anthropologie du développement, vu du Sud et vécu au quotidien
}

\section{Maï Manga Thérèse Keïta}

\section{Q OpenEdition}

\section{Journals}

Édition électronique

URL : http://journals.openedition.org/apad/2743

DOI : 10.4000/apad.2743

ISSN : 1950-6929

Éditeur

LIT Verlag

Édition imprimée

Date de publication : 1 décembre 1993

\section{Référence électronique}

Maï Manga Thérèse Keïta, « Prémices à une anthropologie du développement, vu du Sud et vécu au quotidien », Bulletin de l'APAD [En ligne], 6| 1993, mis en ligne le 10 mars 2008, consulté le 08 septembre 2020. URL : http://journals.openedition.org/apad/2743 ; DOI : https://doi.org/10.4000/ apad. 2743

Ce document a été généré automatiquement le 8 septembre 2020.

Bulletin de l'APAD 


\title{
Prémices à une anthropologie du développement, vu du Sud et vécu au quotidien ${ }^{1}$
}

\author{
Maï Manga Thérèse Keïta
}

1 Travaillant depuis exactement douze ans dans le secteur du développement, domaine de la Sociologie, nous n'avons pourtant jamais considéré et encore moins conçu notre activité comme s'exerçant dans un domaine de la recherche pouvant, un jour ou l'autre (ce jour est peut-être arrivé), constituer une somme, un champ cohérent et homogène des sciences sociales, ayant son propre faisceau de questionnements, ses concepts, sa terminologie, devenir un champ de connaissance spécifique et prometteur.

2 Le fait d'être parmi vous nous amène à reconsidérer les choses, c'est-à-dire, à admettre que le marché du développement pouvait être sérieux, et capable de sécréter sa propre anthropologie. C'est en ce sens que nous saluons l'initiative de l'APAD.

3 Notre attitude qui peut paraître contradictoire ou surprenante, reflète des nombreuses ambiguïtés du chercheur, "faisant dans la masse d'actions et d'activités que l'on regroupe un peu trop vite, sous le concept générique de développement". Nous ne nous lancerons pas dans le défi à la définition du développement, nous savons tous ce que n'est pas le développement, même si nous ne savons pas toujours ce qu'il est exactement. Nous nous pencherons plutôt sur le rôle et le statut de chercheur, tels que vécus au quotidien.

4 En tant que sociologue nous sommes sollicités pour apporter notre contribution technique à la résolution théorique de certains problèmes que rencontrent les initiateurs, concepteurs, gestionnaires et bailleurs de fonds de projets de développement.

5 Ces demandes d'études, ou d'enquêtes socio-économiques sont rarement le fait de populations cibles, que nous distinguons des promoteurs de projets. Tout d'abord, permettez-nous de rappeler que le développement est un marché, comme tous les autres. 
6 Il met en circulation des biens, des marchandises et des moyens financiers. Il possède ses débouchés (les pays à développer), ses financiers (les bailleurs de fonds), son marché de l'emploi (experts internationaux et locaux, employés et agents de toute sorte), il génère des profits, même si la nature et le volume de ses profits restent aussi difficiles à identifier qu'à quantifier, à partir du Sud !

7 Le marché du développement a néanmoins la particularité d'être hautement politique. Les enjeux politiques y sont d'autant plus importants que souvent inavoués et surtout inconnus des populations cibles, tout comme de celles du Nord.

8 Il est réglé selon la division internationale du travail, et reflète avec plus ou moins de fidélité et d'acuité les rapports Nord-Sud, bien qu'enrobés sous l'enveloppe de "l'aide".

Dans le marché du développement, c'est le Nord qui généralement veut aider le Sud, non point pour l'assujettir, ni l'exploiter, mais pour l'aider à être comme lui : développé.

Alors campons notre décor :

11 Le Nord : il est riche, savant, généreux et plein de bonnes intentions. Il ne veut que du bien au Sud. Il est prêt à donner beaucoup: ses experts civils et militaires, ses équipements, ses gadgets, ses modèles de société et de développement, son argent : 0,1 à $0,7 \%$ de son PNB.

12 Le Sud : il est pauvre, ignorant, sale, déchiré par les guerres fratricides, dites guerres tribales, il est affamé et insatiable. Il en demande plus et encore.

Depuis plus de trente ans que le Nord l'aide, le porte à bout de bras, il n'arrive pas à s'en sortir. Bien au contraire, plus en l'aide, plus on a l'impression qu'il régresse et s'enfonce. Le Nord est blanc. Le Sud est noir, jaune, métis, c'est-à-dire africains et assimilés, asiatiques, arabes.

14 Il y a aussi une donnée fondamentale : le Nord fut et demeure colonisateur, le Sud fut et reste essentiellement colonisé. Ceux du Sud, qui ont pu et su s'échapper du camp de concentration de la pauvreté, de la misère sociale et du racisme, sont ceux là qui ont viré la cutie du colonialisme, qui se sont débarrassés du complexe et des réflexes de colonisé pour parler d'égal à égal avec le Nord et, dans certains secteurs, de supérieur à inférieur.

15 Aujourd'hui, disons-le franchement, le Sud est essentiellement noir et arabe, quoique, à la faveur de l'éclatement du bloc socialiste, un nouveau sud se profile dangereusement à l'Est du Nord. Cette impression d'incapacité du Sud à se développer à donné naissance, en France à un spleen que l'on appelle afro-pessimisme. Où donc se situe le sociologue, quel rôle joue-t-il? Dans le marché du développement, il y a le niveau national ou local, et le niveau international.

16 Les chercheurs du Sud, ou techniciens africains du développement, en particulier les consultants, ont dû s'imposer par la qualité de leurs travaux, avant que de se voir intégrer au marché du développement, plus précisément dans les domaines des études et évaluations de projets, avec en prime le pompeux titre d'expert.

17 Parmi les experts, il y a le local et l'international. Le local, le national, est toujours du Sud l'international généralement du Nord. Si le local peut à l'occasion devenir international, l'international ne sera jamais ou presque jamais un local, au risque de se voir payer de trois à cinq fois moins ses services. 
Car, là réside la vraie différence entre les deux : le positionnement géographique n'est qu'un moyen pour les bailleurs de fonds, de faire des économies d'échelle, en sous payant le local, alors que bien souvent il a été formé dans les universités et écoles du Nord a reçu une formation répondant aux critères et normes du Nord, et que notre propre expérience nous a montré qu'une formation supérieure et des prestations supérieures en qualité sont exigées de l'expert local : une ségrégation flagrante, en fait une sorte de sous expert, donc de chercheur de seconde zone !

Dans les contrats d'étude, il est toujours exigé du chercheur-expert local, de ne point divulguer, ni utiliser les résultats de ses travaux.

L'expert n'intervient que de manière sporadique, sur des questions ponctuelles. Il lui est difficile d'accumuler et de capitaliser les connaissances ou informations recueillies d'un projet à un autre.

Il lui est tout simplement demandé de devenir amnésique, dès remise du rapport de mission.

Il est pratiquement interdit au chercheur, d'utiliser son propre travail pour approfondir ses connaissances, et enrichir la connaissance des milieux étudiés, donc enrichir les sciences sociales au nom desquelles il est sollicité !

Le marché du développement est aussi sexiste. La femme chercheure, expert local est souvent orientée pour ne pas dire confinée à l'étude d'aspects féminins, parce que bailleurs de fonds et décideurs s'accordent pour penser et faire en sorte que seule la femme peut comprendre les problèmes des femmes et communiquer avec.

Ainsi, la chercheure, locale ou internationale, se retrouve enfermée dans le gynécée local et international du développement avec tout ce que cela implique de grandeur morale et de misère technique.

Dans ces conditions, les contradictions propres au statut de chercheur sont nombreuses et elles ont pour effet de l'éloigner de la recherche, pour en faire un bon exécutant de tâches et d'études répétées et répétitives.

A la différence de son homologue local, l'expert international, l'expert du Nord ou assimilé, capitalise à peu de frais et d'effort le produit de ses activités.

$\mathrm{Au}$ cours de brefs mais nombreux séjours dans le Sud, compilant travaux locaux, rapports de séminaires, conversations et discussions anodines, il publiera aisément un ou des ouvrages, sans citer les contributions formelles ou informelles des nationaux. Très vite, grâce aux facilités d'édition, il devient expert ambulant et même volant.

Nous savons que le développement pour s'assurer un minimum de succès demande continuité, persévérance, suivi, ouverture d'esprit, imagination et capacité de se remettre en cause. Mais, nous constatons aussi que le statut et le rôle du chercheur tels qu'imposés par la structure du marché sont en contradiction avec les exigences d'un vrai développement.

29 Alors, sur quoi se fonde la naissance de l'anthropologie du développement? Sur quelles connaissances, quelles pratiques? Quel type de rapport entre le chercheur expert et le chercheur expert international? Nous croyons que l'anthropologie du développement doit précéder le développement, mais, se construira-t-elle en marge de rapports généraux et généralisés de dépendance Nord-Sud?

Quel est son avenir alors même que l'anthropologie africaine d'Afrique n'a toujours pas vu le jour, faute d'enracinement solide dans la longue histoire de notre continent, et 
faute de reconnaître l'apport scientifique des pères africains de l'anthropologie, nous pensons en particulier à Cheikh Anta Diop, devenu aujourd'hui incontournable pour l'ensemble des jeunes chercheurs africains.

31 L'anthropologie africaine, pour créer sa dynamique propre, devra s'obliger non seulement à l'étude de son propre milieu mais aussi porter son regard sur les autres formations sociales, celles d'Europe en particulier, compte tenu des liens de dépendances et d'interférences, et aussi sur l'ensemble des sociétés humaines.

32 Elle devra se libérer de son carcan géographique, politique, culturel et psychologique, en faisant des autres formations sociales non africaines des objets d'étude, pour mieux saisir les fonctionnements de nos sociétés et surtout être capable de projection et de précision indispensables au développement.

33 Niamey, le 19 octobre 1992

\section{NOTES}

1.L'auteur, qui avait présenté une communication aux Journées APAD d'octobre 1992 à Montpellier, a souhaité que le Bulletin fasse état de ce texte. 